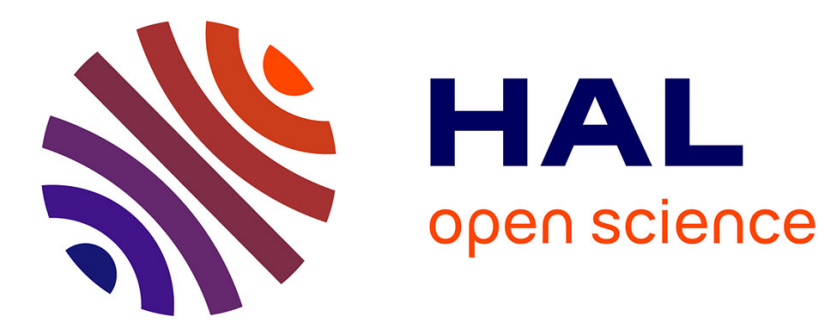

\title{
Common tools for quantitative pulse and step-heating thermography - Part I: theoretical basis
}

\author{
D. Balageas, J.M. Roche
}

\section{To cite this version:}

D. Balageas, J.M. Roche. Common tools for quantitative pulse and step-heating thermography Part I: theoretical basis. The 12th International Conference on Quantitative InfraRed Thermography (QIRT 2014), Jul 2014, BORDEAUX, France. 10.1080/17686733.2014.891324 · hal-01132100

\author{
HAL Id: hal-01132100 \\ https://hal.science/hal-01132100
}

Submitted on 16 Mar 2015

HAL is a multi-disciplinary open access archive for the deposit and dissemination of scientific research documents, whether they are published or not. The documents may come from teaching and research institutions in France or abroad, or from public or private research centers.
L'archive ouverte pluridisciplinaire HAL, est destinée au dépôt et à la diffusion de documents scientifiques de niveau recherche, publiés ou non, émanant des établissements d'enseignement et de recherche français ou étrangers, des laboratoires publics ou privés. 


\section{Common tools for quantitative pulse and step-heating thermography - Part I: theoretical basis}

D. Balageas *, J.M. Roche

The 12th International Conference on Quantitative InfraRed Thermography (QIRT 2014) BORDEAUX, FRANCE

7-11 juillet 2014 

Common tools for quantitative pulse and step-heating thermography - Part I: theoretical basis

Outils communs pour le traitement quantitatif de données de thermographie impulsionnelle et en créneau - Partie I : bases théoriques

\section{par}

D. Balageas *, J.M. Roche

* Univ. Bordeaux, I2M, TREFLE Dept., Talence, France

\section{Résumé traduit :}

Différents outils de pré- et post-traitement avancé ont été développés au cours des deux dernières décennies, dans le but d'optimiser les performances du CND par thermographie impulsionnelle, en terme de détection et de caractérisation de défauts. Deux des techniques les plus efficaces sont la TSR (Thermographic Signal Reconstruction), proposée par Shepard et al. en 2001 et ayant récemment bénéficié d'un nouveau développement basé sur les images de coefficients (Roche et al., 2014), et la détection précoce à contraste émergent, proposée par Krapez et al. en 1994. L'enjeu du travail est ici d'illustrer comment ces outils, communément employés pour la thermographie impulsionnelle, peuvent également être appliqués à l'excitation thermique de type "créneau".

L'étude est divisée en deux parties : la partie analytique théorique fait l'objet du présent article ; les résultats expérimentaux correspondant sont discutés dans un second article. 



\title{
Common tools for quantitative pulse and step-heating thermography - Part I: theoretical basis
}

\author{
by Daniel L. Balageas*, Jean-Michel Roche** \\ * Univ. Bordeaux, I2M, TREFLE Dept., UMR 5295, F-33400 Talence, France, daniel.balageas@wanadoo.fr \\ ** ONERA, Composite Materials and Structures Dept., BP72, 92322 Châtillon cedex, France
}

\begin{abstract}
Several advanced pre- and post-processing tools have been developed over the last two decades, to enhance the performances of pulse thermography, in terms of defect detection and characterization. Two of the most efficient techniques are the Thermographic Signal Reconstruction (TSR), proposed by Shepard et al. in 2001, including a recent development based on the use of the polynomial coefficient images proposed by Roche et al. in 2014, and the early detection at emerging contrast, proposed by Krapez et al. in 1994. The stake of this work is to show how these tools, commonly used for pulse-heating, can be applied to step-heating.
\end{abstract}

The work was divided in two parts: the theoretical and analytic study is reported in the present article; the matching experimental results are discussed in a second, separate article.

\section{Introduction}

Time-resolved thermographic techniques for non-destructive evaluation (NDE) are varied, depending on the type of thermal stimulations: short-pulse (Dirac), step-heating, squared-shaped-heating. For most applications, the pulse technique is used [1-4]. It offers many advantages (rapid measurement, simple set-up, easy physical interpretation). This is why several tools have been developed to improve the pre- and post-processing of thermal data and images, such as the Thermographic Signal Reconstruction (TSR) [5-7] and the early detection/characterization method, based on emerging contrast [8-10]. The principles of both methods have been previously presented and illustrated in separate publications devoted to pulse thermography. The application of these processing tools to step-heating and squareshaped heating (which can be regarded as the succession of a "positive" step-heating and a "negative" one) could give more attractiveness to these techniques, which, although less popular than pulse-heating, remain a possible alternative [11-14] to detect deep defects and a way to decrease the maximum temperature reached by the tested samples. Until now, very few attempts of such application have been made and without any theoretical basis [15].

In this context, the aim of the present work is to justify and illustrate the application of these tools to stepheating. In particular, our study proposes to theoretically (Part I, present article) and experimentally (Part II, companion article [16]) investigate the potential of the TSR and early detection methods for step-heating thermography and to compare the results with those of pulse-heating.

\section{Analytic modeling of front-face step-heating thermograms}

In this section, the front-face temperature increase $\Delta T$, resulting from a step-heating excitation, is analytically modeled in the four following "standard" configurations:

- $\quad$ semi-infinite medium, with no defect;

- $\quad$ slab of thickness $L$, with no defect;

- $\quad$ slab with a defect of infinite thermal resistance, embedded at a depth $z_{d}=L$;

- $\quad$ slab with a defect of finite thermal resistance, embedded at a depth $z_{d}=L$.

For each configuration, the medium is supposed to be homogeneous and adiabatic; as for the defect, its lateral dimensions are supposed to be large enough to avoid 2D or 3D effects.

\subsection{Semi-infinite medium with no defect}

The temperature increase of the front-face of a semi-infinite medium (or a thermally thick slab), caused by a step-heating, is given by the simple Equation (1):

$$
\Delta T=\frac{2 q}{e} \cdot \frac{\sqrt{t}}{\sqrt{\pi}}
$$

where $q$ is the heat flux density, e the material effusivity and $t$ the time. Consequently, in a log-log diagram, the associated curve is a straight line of slope $+1 / 2$. 


\subsection{Slab with no defect}

In the case of a slab of finite thickness $L$, the front-surface temperature increase is given by:

$$
\Delta T=\frac{2 q}{e} \cdot \frac{\sqrt{t}}{\sqrt{\pi}}\left[1+\sqrt{\pi} \sum_{n=1}^{\infty} 2 \operatorname{ierfc}\left(\frac{n}{\sqrt{\kappa t} / L}\right)\right]
$$

where ierfc is the integral of the complementary error function erfc, and $\kappa$ the thermal diffusivity. A non-dimensional form of this equation is:

$$
\overline{\Delta T}=\frac{\Delta T}{q L / k}=2 \frac{\sqrt{F o_{L}}}{\sqrt{\pi}}\left[1+\sqrt{\pi} \sum_{n=1}^{\infty} 2 \operatorname{ierfc}\left(\frac{n}{\sqrt{F_{O L}}}\right)\right]
$$

where $k$ is the material thermal conductivity and $F_{O_{L}}$ the Fourier number relative to the thickness $L: F_{O_{L}}=\kappa t / L^{2}$. This expression can also be written as:

$$
\overline{\Delta T}=\frac{\Delta T}{q L / k}=2 \frac{\sqrt{F o_{L}}}{\sqrt{\pi}}\left\{1+2 \sum_{n=1}^{\infty}\left[\exp \left(-\frac{n^{2}}{F o_{L}}\right)-\frac{n \sqrt{\pi}}{\sqrt{F O_{L}}} \operatorname{erfc}\left(\frac{n}{\sqrt{F O_{L}}}\right)\right]\right\}
$$

\subsection{Slab with defect of infinite thermal resistance}

The solution for a large-extent defect of infinite thermal resistance embedded at a depth $z_{d}$ in a semi-infinite medium or in a slab is identical to the one obtained for a homogeneous slab. In this case, Equations (2-2") are relevant for the defective zone, replacing the slab thickness $L$ by the defect depth $z_{d}$ and the Fourier number $F_{O_{L}}=\kappa t / L^{2}$ by $F O_{d}=\kappa t / z_{d}^{2}$.

The relative contrast $C_{r}$ created by the defect is then deduced from Eq. $(1,2)$ for short times (early contrast):

$$
C_{r}=\frac{\Delta T_{d}-\Delta T_{\infty}}{\Delta T_{\infty}}=2 \sqrt{\pi} \sum_{n=1}^{\infty} \operatorname{ierfc}\left(\frac{n}{\sqrt{F o_{d}}}\right)
$$

or from Equation (2) for any time:

$$
C_{r}=\frac{\Delta T_{d}-\Delta T_{L}}{\Delta T_{L}}=2 \sqrt{\pi}\left[\frac{\sum_{n=1}^{\infty} \operatorname{ierfc}\left(n / \sqrt{F o_{d}}\right)-\sum_{n=1}^{\infty} \operatorname{ierfc}\left(n / \sqrt{F o_{L}}\right)}{1+2 \sqrt{\pi} \sum_{n=1}^{\infty} \operatorname{ierfc}\left(n / \sqrt{F o_{L}}\right)}\right]
$$

Limiting the studied time-domain to relative contrasts lower than $5 \%$, expression (3) applies, and, by analogy with the asymptotic expression $\left(R_{d}=\infty\right)$ proposed for pulse-heating [3,14],

$$
C_{r} \approx 2 \exp \left(-\frac{1}{F o_{d}}\right)
$$

the relative contrast resulting from a step-heating excitation can be approximated by Eq. (5):

$$
C_{r} \approx 0.470 \exp \left(-\frac{1.225}{F o_{d}}\right)
$$

\subsection{Slab with defect of finite thermal resistance}

For a slab with a large-extent defect of finite normalized thermal resistance $R_{d}^{*}=z_{d} R_{d} / k, z_{d}$ and $R_{d}$ being respectively its depth and thermal resistance, and $k$ the slab thermal conductivity, a solution can be obtained using the analytical solution proposed in [2] or the quadrupole method [13]. With this approach, followed by [4, 11], the emerging contrast (relative contrast lower than $5 \%$ ) created by the defect is seen as the difference between the solution of the defective slab, $\Delta T_{d}$, and the solution of the semi-infinite medium with no defect, $\Delta T_{s}$ - Equation (1).

Similarly to the expression adopted for pulse-heating [6, 8], reminded by Equation (6):

$$
C_{r} \approx 2 \exp \left(-\frac{1}{F o_{d}}\right)\left[1-2 \frac{F o_{d}}{R_{d}^{*}+2 F o_{d}}\right]
$$

an analytical approximation can be given as well for step-heating, for a finite thermal resistance (Equation (7)):

$$
C_{r} \approx 0.470 \exp \left(-\frac{1.225}{F o_{d}}\right)\left[1-\frac{\sqrt{F o_{d}}}{R_{d}^{*}+\sqrt{F o_{d}}}\right]
$$




\section{Relevance of the TSR processing for step-heating}

\subsection{TSR technique: recall of the basics}

As theorized by Schenck [17] and recalled in [3], the "rectification" ${ }^{1}$ of a curve, i.e. making a part of it linear, is very useful since it defines a domain "anticipated by theoretical expectations and provides confirmation by slope measurements that theory and experiments are in fair agreement" [17]. For pulse thermograms, this rectification is obtained by plotting the temperature rise $\Delta T$ as a function of time $t$ in a log-log diagram: following a thermal pulse, the surface temperature decay of a semi-infinite medium (or its first part in the case of a slab) becomes a straight line, with a $-1 / 2$ slope, indicating that the 1-D diffusion law is applicable (Fig. 1a). In the case of a damaged medium, the thermograms associated with its sound zone follow this linear behavior, while the ones associated with the local defects deviate from it after a certain time, which makes their detection possible.

The described linear behavior, observed in a log-log plot, justifies the idea to perform a logarithmic polynomial global fitting of the thermograms:

$$
\log _{10}(\Delta T)=a_{0}+a_{1} \log _{10}(t)+a_{2}\left[\log _{10}(t)\right]^{2}+\ldots+a_{n}\left[\log _{10}(t)\right]^{n}
$$

Their logarithmic differentiations, which are the basis of the TSR method, not only provide a better signal-tonoise ratio (SNR) to pulse thermographic NDE and enhance defect detectability, but also allow a drastic data compression.

In the present work, the possibility to apply the TSR technique to step-heating thermography is theoretically established in the case of a slab without defect or with a defect of infinite thermal resistance. A comparison with pulse thermography is made, based on the criteria of defect detectivity and detection precociousness, two key-parameters related to the SNR and sharpness of the defect images.

\subsection{Application of TSR to step-heating}

The rectification of the front-face step-heating thermograms is achieved by their plotting in a log-log scale, similarly to what is done for pulse-heating.

Figure 1 illustrates the typical normalized thermograms for both heat deposition types: as expected, two asymptotes are found for pulse-heating (-1/2 slope for short times and null-slope for late times), as well as for stepheating (+1/2 slope for short times and +1 slope for late times).
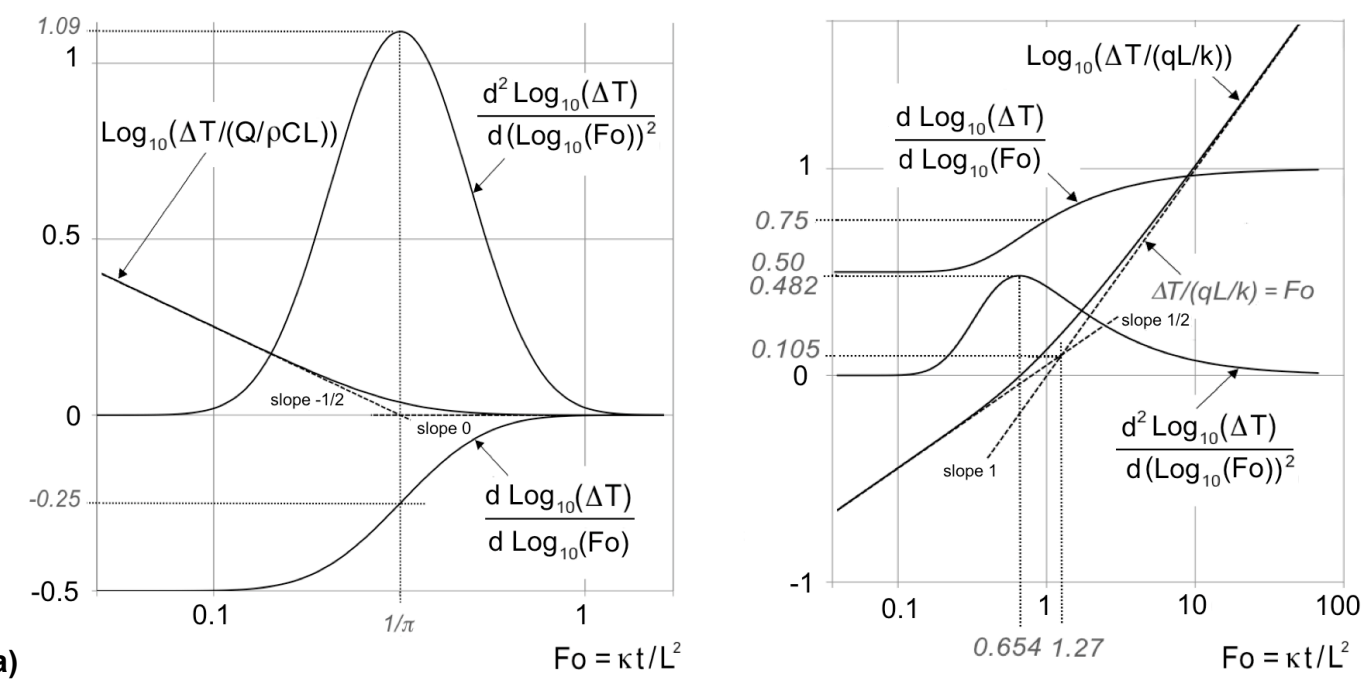

(b)

Fig. 1. Similarities of the thermograms and logarithmic derivatives in the case of a slab of thickness $L$ submitted to pulse(a) and step- (b) heating. Same results would be observed for an infinite thermal resistance defect embedded at a depth $z_{d}=L$ in a semi-infinite medium or in a slab.

Let us note that, due to the difficulty to have a reliable and accurate knowledge of the parameters of the experiment (heat flux density $q$ for step-heating; fluence $Q$ for pulse-heating) and of the sample characteristics (thickness $L$, thermal conductivity $k$ and thermal diffusivity $\kappa$ ), the thermograms can simply be normalized by one value taken shortly after the beginning of the heat deposition to mitigate the non-uniform heat deposition. Such an operation is easy for pulse-heating but problematic for step-heating because of the very low SNR of the thermal signal at that moment. The logarithmic derivatives, on the contrary, are automatically normalized.

\footnotetext{
${ }^{1}$ The meaning of the term "rectification" is here different from the classic one used in mathematics for the determination of the length of an irregular arc segment.
} 
For both types of stimulation, the $F_{O_{L}}$ domain relevant for NDE purposes (i.e. relevant to assess parameters such as thickness and defect depth) is the transition between the asymptotes. Indeed, for low and high Fourier numbers, the sensitivity to the defect is negligible. The extent of this domain of interest is much larger for step-heating than for pulse-heating, as it can be seen in Fig. 1 and Table 1. This makes step-heating pertinent for thick materials and deep defects. However, this domain occurs later for step-heating, which is a drawback, since it favors the data contamination by 3D thermal diffusion effects (blurred images).

\begin{tabular}{cccc}
\hline \multirow{2}{*}{$\begin{array}{c}\text { Type of } \\
\text { experiment }\end{array}$} & \multicolumn{2}{c}{ Maximum of contrast } & \multirow{2}{\text{ExtensionofFodomain}}{$\begin{array}{c}\text { usable for thickness } \\
\text { defect depth identification }\end{array}$} \\
\cline { 2 - 3 } Pulse-heating & 0.5 & Second derivative & $0.16-<1$ \\
Step-heating & 0.5 & 1.09 & $0.3->10$ \\
\hline Step/Pulse ratio & 1.0 & 0.482 & $>11.5$ \\
\hline
\end{tabular}

Table 1. Comparison between pulse- and step-heating potential for thickness or defect depth identification by methods based on logarithmic derivatives exploitation (TSR method).

Let $\overline{\Delta T^{\prime}}$ and $\overline{\Delta T^{\prime \prime}}$ be the first and second logarithmic derivatives of the thermogram $\Delta T$. To compute them, a simpler analytical expression of $\overline{\Delta T}$ [18-20] can be used:

$$
\overline{\Delta T}=\frac{\Delta T}{q L / k}=\frac{1}{3}+F_{o}-2 \sum_{n=1}^{\infty} \frac{\exp \left(-n^{2} \pi^{2} F_{o}\right)}{n^{2} \pi^{2}}
$$

leading to the following expressions of $\overline{\Delta T^{\prime}}$ and $\overline{\Delta T^{\prime \prime}}$ :

$$
\begin{gathered}
\overline{\Delta T^{\prime}}=\frac{\mathrm{d}\left[\log _{10}(\Delta T)\right]}{\mathrm{d}\left[\log _{10}(F o)\right]}=F_{o}\left[1+2 \sum_{n=1}^{\infty} \frac{\exp \left(-n^{2} \pi^{2} F o\right)}{\overline{\Delta T}}\right] \\
\overline{\Delta T^{\prime \prime}}=\frac{\mathrm{d}^{2}\left[\log _{10}(\Delta T)\right]}{\mathrm{d}\left[\log _{10}(F o)\right]^{2}}=2.30216 F_{o}\left[\overline{\Delta T^{\prime}}\left(1-\overline{\Delta T^{\prime}}\right)-2 F_{o} \sum_{n=1}^{\infty} n^{2} \pi^{2} \frac{\exp \left(-n^{2} \pi^{2} F o\right)}{\overline{\Delta T}}\right]
\end{gathered}
$$

The time-evolutions of these derivatives are plotted in Figure 1 and compared to the ones for pulse-heating, taken from [21]. Pulse- and step-heating curves are similar, at least at the beginning of the transition period when an early detection can be achieved. For sake of comparison, the step-heating derivative curves are shifted along the $y$-scale (Fig. 2), in order to point out the following similarities with pulse-heating:

- $\quad$ the amplitude of variation of the first derivative is the same in both step- and pulse-heating;

- $\quad$ the time-evolution of the rising part of the step-heating second derivative seems to be very well approximated by a Gaussian, which was demonstrated in [21, 22] for pulse-heating (for the whole curve).
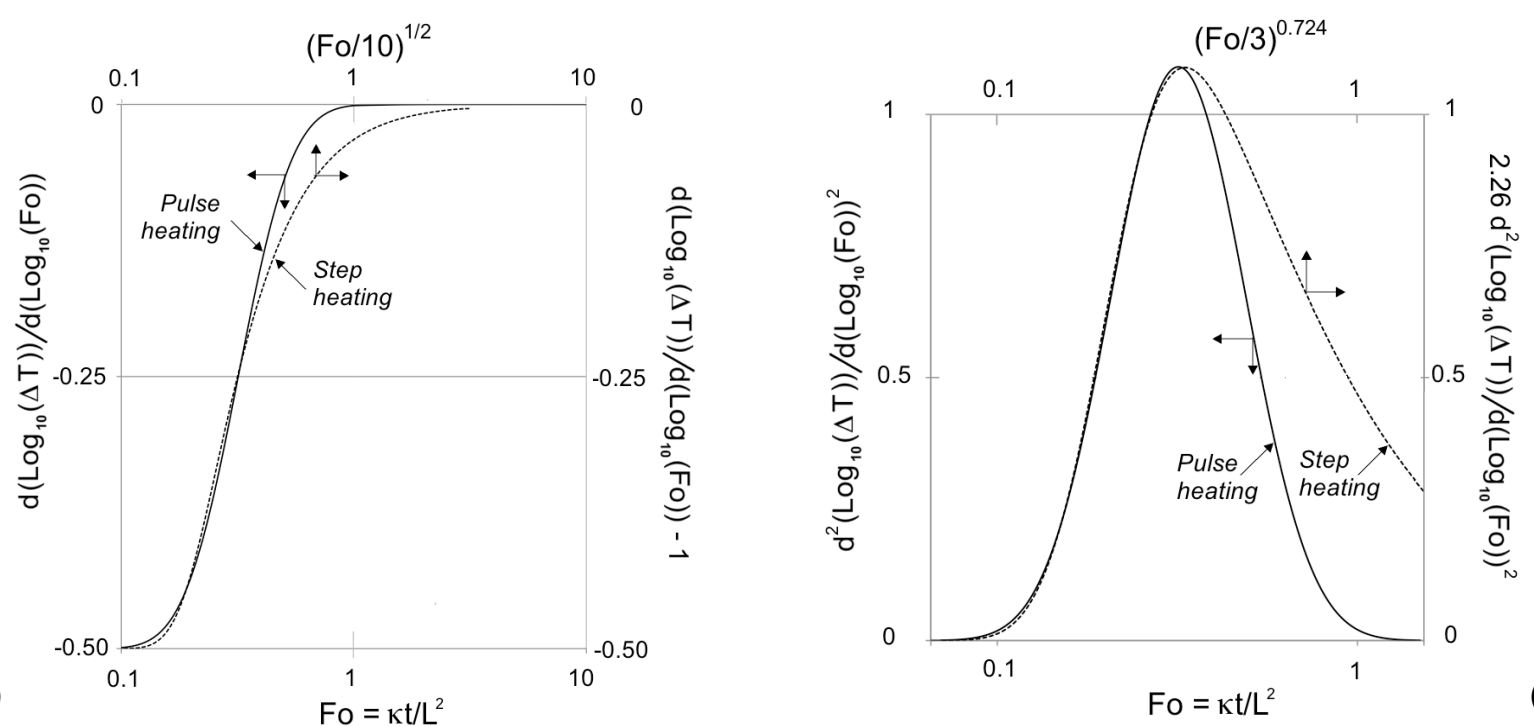

(b)

Fig. 2. Analogy of the shapes of (a) the first and (b) second logarithmic derivatives for pulse- and step-heating (same case as for Fig. 1). 
On the other hand, several differences with pulse-heating can still be observed:

- for step-heating, the parameter governing the evolution of the first logarithmic derivative is not, as a first approximation, the Fourier number, but its square root, which has to be linked to the expression describing the contrast generated by defects (see Eq. (7));

- the three remarkable points defined as (i) the intersection of the two asymptotes of the thermograms, (ii) the half-rise of the first derivative and (iii) the maximum of the second derivative, do not occur for the same Fourier number anymore;

- $\quad$ the amplitude of the second derivative maximum is lower for step-heating, which must lead to a lower defect detectivity.

From Figure 3, the "early" times, associated with a $1 \%$ relative contrast on the thermograms and on the logarithmic derivatives, can be assessed for pulse- and step-heating. As for early detection matters, it appears that the "precession of the emerging contrast" due to the successive differentiations, described in [3, 21] for pulse-heating, is also observed for step-heating. The values of these early times are displayed in Table 2 and compared to the characteristic times (asymptote intersection of thermogram, first derivative half-rise and second derivative maximum). The ratios of these parameters, which can be considered as image sharpness indicators, are computed: they show that pulse-heating is always more satisfactory than step-heating, no matter what type of image is considered (temperature, first or second logarithmic derivative) and what approach is chosen (imaging and identification at characteristic points or early detection and characterization based on emerging contrast).
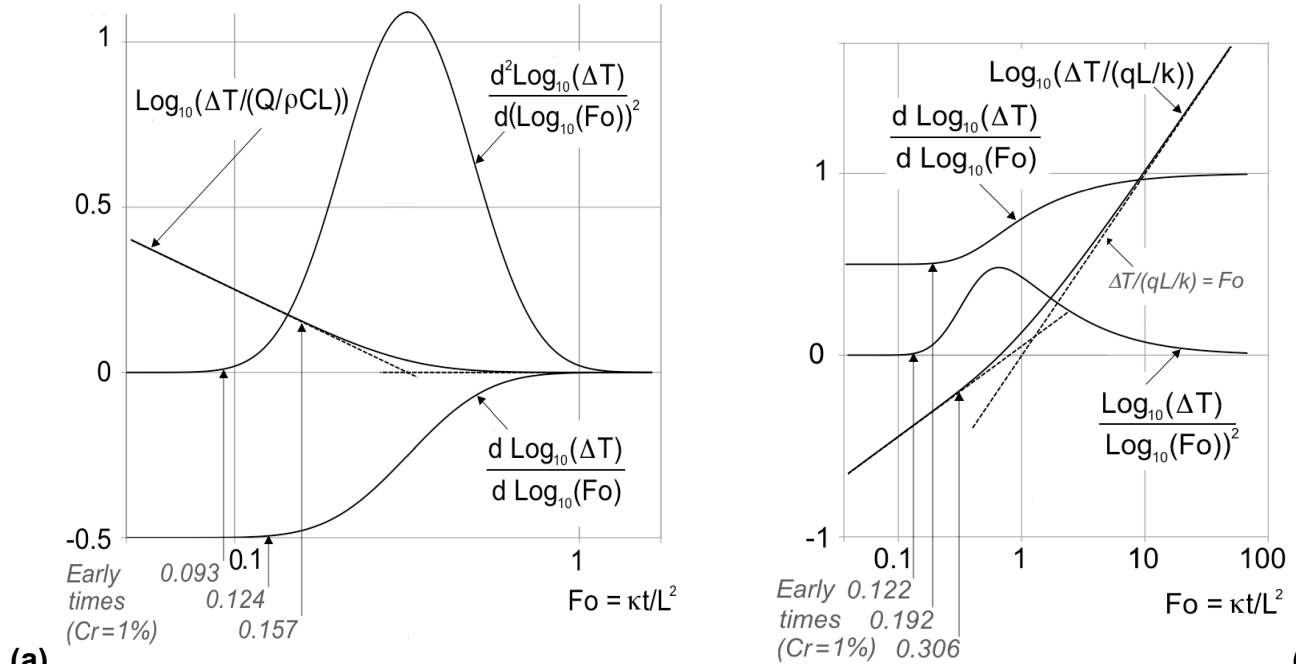

(b)

Fig. 3. "Precession" of the early defect detection due to successive differentiations. Comparison between pulse- (a) and step- (b) heating for the thermograms and their logarithmic derivatives (same case as for Fig. 1).

\begin{tabular}{ccccccc}
\hline & \multicolumn{5}{c}{$\begin{array}{c}\text { Detection precociousness } \\
\text { Type of } \\
\text { experiment }\end{array}$} & \multicolumn{3}{c}{$\begin{array}{c}\text { Early detection approach } \\
(F o \text { for 1\% of relative contrast })^{\star}\end{array}$} \\
\cline { 2 - 7 } & $\begin{array}{c}\text { Thermogram } \\
\text { asymptotes } \\
\text { intersection }\end{array}$ & $\begin{array}{c}\text { First } \\
\text { derivative } \\
\text { half-rise }\end{array}$ & $\begin{array}{c}\text { Second } \\
\text { derivative } \\
\text { maximum }\end{array}$ & Thermogram & $\begin{array}{c}\text { First } \\
\text { derivative }\end{array}$ & $\begin{array}{c}\text { Second } \\
\text { derivative }\end{array}$ \\
\hline Pulse-heating & 0.318 & 0.318 & 0.318 & 0.157 & 0.124 & 0.093 \\
Step-heating & 1.27 & 1.000 & 0.654 & 0.306 & 0.192 & 0.122 \\
\hline $\begin{array}{c}\text { Sharpness ratio } \\
\text { (step/pulse) }\end{array}$ & 0.250 & 0.318 & 0.486 & 0.513 & 0.646 & 0.762 \\
\hline
\end{tabular}

* For the the derivatives, it is $1 \%$ of the maximum of the relative contrast which is considered.

Table 2. Comparison between pulse- and step-heating potential for thickness or defect depth identification by the logarithmic derivatives exploitation coupled with the early detection approach. Values are taken from the analysis of the thermograms and their logarithmic derivatives (Fig. 1 and 3).

The next step consists in evaluating the contrasts created by a defect, both for pulse-heating (from Eq. $(4,6)$ ) and step-heating (exact values and approximated ones, deduced from Eq. (5,7)). Their time-evolutions are compared in Fig. 4: in both cases, the influence of the thermal resistance decreases when the contrast tends to zero. Nevertheless, 
this convergence towards the asymptotic case $\left(R_{d} \rightarrow \infty\right)$, remarkable for pulse-heating, is not very pronounced for stepheating. This will have a negative influence on the accuracy of the identification of the defect depth by the early detection approach, as it will be shown in the next section.

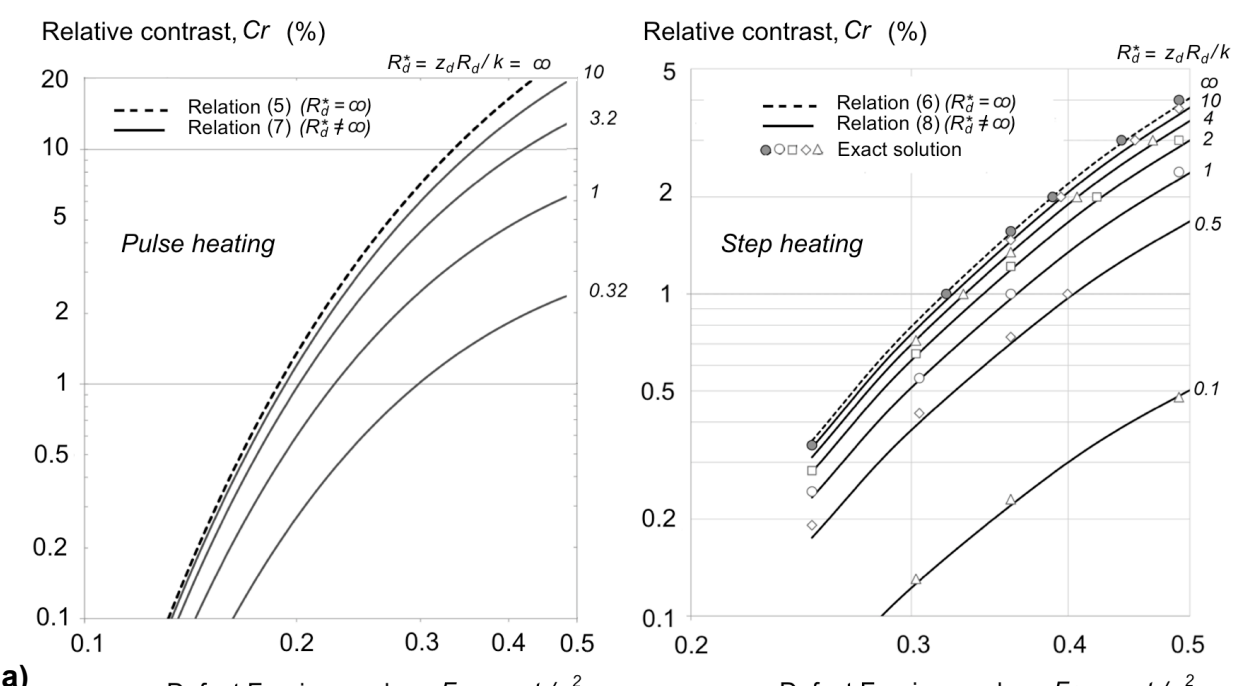

(a)

Defect Fourier number, $F o_{d}=\kappa t / z_{d}^{2}$

(b)

Fig. 4. Time-evolution of the emerging relative contrast for various defects of normalized thermal resistance $R_{d}^{*}$, embedded at depth $z_{0}$ (a) pulse-heating; (b) step-heating.

\subsection{Discussion}

The limits of this theoretical study are twofold: first, the slabs are supposed perfectly adiabatic; second, only the case of an infinite thermal resistance is considered for the study of the derivatives. In reality, this resistance may vary considerably, which would have an influence on the results presented in Table 2. However, at least as a first approximation, the trends deduced in this article can be considered as realistic.

The influence of the defect thermal resistance on the temperature rise contrast has been studied, showing a behavior of the step-heating thermograms slightly different from the one of pulse-heating thermograms. This will have an influence on the accuracy of the identification of the defect parameters $\left(z_{d}\right.$ and $\left.R_{d}\right)$, as it will be shown in the next sections.

The TSR operations (thermogram fitting by a logarithmic polynomial, logarithmic differentiations and subsequent detection precession) lead to analogous results for both pulse- and step-heating, which allows us to assume that the TSR technique is applicable to step-heating and will lead to similar results, in particular for:

defect detectivity enhancement by use of derivative images;

- $\quad$ all-defect imaging by a unique, composite image built from the fitting logarithmic polynomials coefficients [7].

These conclusions and assumptions are experimentally discussed in Part II of this article [16].

\section{Relevance of the early detection approach for step-heating}

The early detection/identification concept has been applied not only in the NDE domain, but also in the field of thermophysics [10]. For thermographic NDE, it has been proposed at the beginning of the nineties [8, 9]. Nevertheless, it has been scarcely applied, mainly because of the poor thermal sensitivity of the infrared cameras that were available at that time and because of the complexity of certain procedures to practically apply the idea. That explains why, a few works excepted, most of the thermography studies kept on focusing on the contrast SNR issue and were therefore based on the maximum contrast approach.

Today, the high sensitivities and high frame rates reached by infrared cameras make the quantitative use of the emerging contrast reliable. The use of the TSR method as a data pre-processing, leading to excellent signal-to noise ratios (SNRs), gives even more credit to the technique.

\subsection{Early detection approach: recall of the basics}

The early detection approach relies on the following six operations:

- $\quad$ step 1: choice of a model, generally 1D, simpler than the actual configuration;

- $\quad$ step 2: choice of an early-time window for the analysis of the thermograms, so that very few parameters be influent (ideally only one); 
- $\quad$ step 3: solving of the inverse problem in these conditions, preferentially using explicit relations;

- $\quad$ step 4: analysis of the evolution with the contrast of the so-identified parameter, for the assessment of its accuracy;

- $\quad$ step 5: choice of a fitting function for this evolution;

- step 6: extrapolation to zero-contrast to make the most precise estimation of the parameter.

The application of this procedure to pulse thermography has been described in details and the intrinsic accuracy of the identification of the defect depth and thermal resistance has been evaluated in [3]. The same method is presently applied to step-heating and the results compared to the ones taken from [3].

\subsection{Application of early detection to step-heating}

For emerging contrast $\left(1 \% \leq C_{r} \leq 5 \%\right)$, a simple expression of the defect depth $z_{d}$ can be deduced from Eq. (5):

$$
z_{d}=0.903 \sqrt{\kappa t \ln \left[\frac{0.470}{C_{r}(t)}\right]}
$$

This expression is valid only for an infinite thermal resistance (asymptotic law $R_{d} \rightarrow \infty$, see Fig. 4b) and must be considered as a first-order approximation. It is analogous to the one found for pulse-heating:

$$
z_{d}=\sqrt{\kappa t \ln \left[\frac{2}{C_{r}(t)}\right]}
$$

The defect depth can be evaluated for various values of the emerging contrast $C_{r}\left(t_{i}\right)$, the choice of the contrast threshold depending on the noise of the thermogram. The so-identified defect depths $z_{d}\left(C_{r}\right)$ suffer from a bias due to the fact that the actual thermal resistance of the defect is not infinite: the lower the thermal resistance, the higher the error. Similarly to what was previously shown for pulse-heating in [3] (Fig. 5a), Fig. 5b shows that, for emerging contrasts of a few percents, the error is a quasi-linear function of the relative contrast. For a few values of contrast, it is then possible to evaluate the depth and to perform a linear extrapolation to zero-contrast, which leads to the best possible estimate of $z_{d}$ : $z_{d}(\mathrm{Cr}=0)$. Errors on the extrapolated $z_{d}$ value do not exceed a few percents (less than $4 \%$ for $R_{d}^{*}=1$ ).
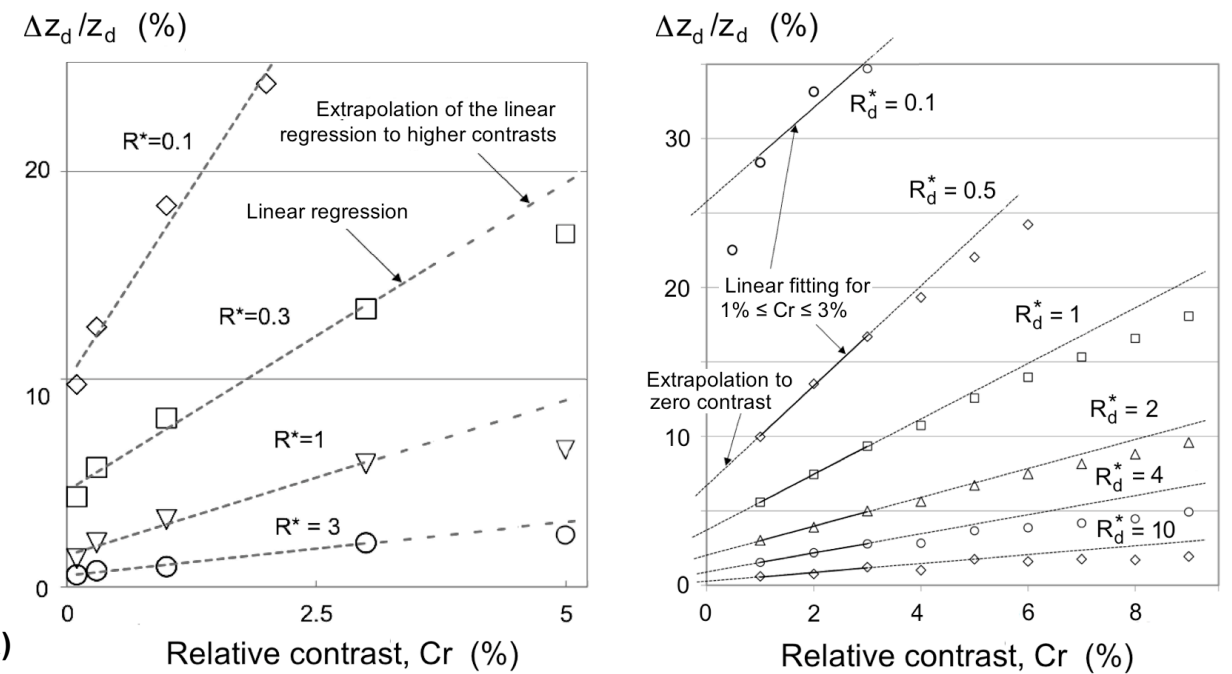

(b)

Fig. 5. Accuracy of the identified defect depth: illustration of the benefit to linearly extrapolate to zero-contrast. (a) pulseheating, using the $1^{\text {st }}$-order expression (13); (b) step-heating, using the $1^{\text {st }}$-order expression (12).

The comparison between pulse- and step-heating is in favor of pulse-heating, the extrapolated values of $z_{d}$ being more precise in this latter case. It is confirmed by considering the empirical relations that can be deduced between the error and the normalized thermal resistance:

- for step-heating:

$$
\log _{10}\left(\frac{\Delta z_{d}}{z_{d}}\right)_{C r \rightarrow 0}=0.54-0.89 \log _{10}\left(R_{d}^{*}\right)
$$

- for pulse-heating:

$$
\log _{10}\left(\frac{\Delta z_{d}}{z_{d}}\right)_{C r \rightarrow 0}=0.20-0.83 \log _{10}\left(R_{d}^{*}\right)
$$


As illustrated in Fig. 6, the identified defect depth at zero-contrast is 2.2 times more accurate with the pulseheating than with the step-heating. It should also be noted that apart from enhancing the intrinsic accuracy of the identification, the extrapolation procedure also enhances its precociousness, which reduces the effects of lateral heat transfer.

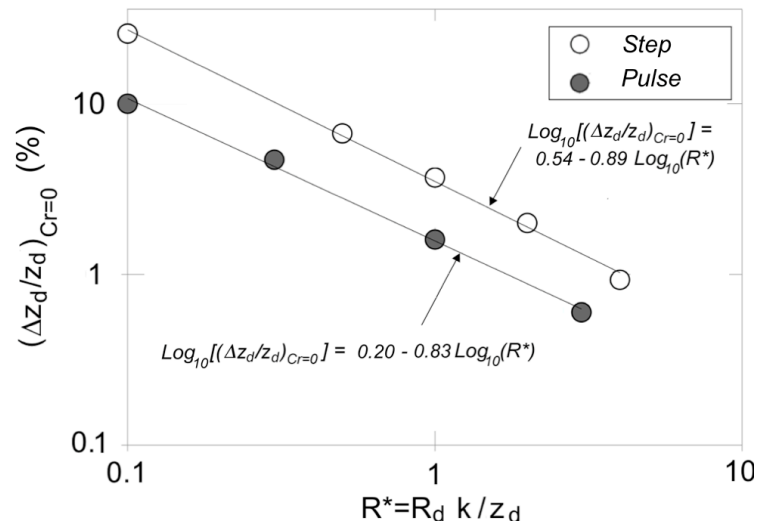

Fig. 6. Errors on the defect depth identified from the emerging contrast, using the asymptotic relation (12) and a linear fitting followed by extrapolation to zero-contrast for step-heating and comparison to the same approach applied to pulseheating, using relation (13).

Once the depth is estimated, it is possible to identify the normalized thermal resistance $R_{d}^{*}$ using the following expression derived from the second-order Eq. (7) in which the Fourier number is evaluated from the extrapolated defect depth value $z_{d}(C r=0)$ :

$$
R_{d}^{*}=\frac{C_{r}(t) \sqrt{F o_{d}}}{0.47 \exp \left(-1.225 / F o_{d}\right)-C_{r}(t)}
$$

The thermal resistance can then be deduced:

$$
R_{d}=R_{d}^{*} \frac{z_{d}}{k}=\frac{C_{r}(t) \sqrt{t}}{e} \cdot \frac{1}{0.47 \exp \left[-1.225 /\left(\left.\kappa t z_{d}^{2}\right|_{C r \rightarrow 0}\right)\right]-C_{r}(t)}
$$

For this identification of the thermal resistance, experimental values of the contrast have to be chosen slightly higher than the ones used for the depth identification (since the contrast is not influenced by the thermal resistance for short times), but not too much to minimize the $3 \mathrm{D}$ effects. In order to better define the optimal conditions for the thermal resistance identification, the accuracy on the identified value of $R_{d}^{*}$ has been evaluated using formula (16) for various $F_{o_{d}}$, and relative contrast $C r$. Here, the relative error on $R_{d}^{*}, \Delta R_{d}^{*} / R_{d}^{*}$, is due to the error on the defect depth, $\left(\Delta z_{d}^{*} / z_{d}^{*}\right)_{C r \rightarrow 0}$, assuming that the value $\left(\Delta z_{d}^{*} / z_{d}^{*}\right)_{C r \rightarrow 0}$ is not corrected using Eq. (14). So the present evaluation of $\Delta R_{d}^{*} / R_{d}^{*}$ must be considered as very pessimistic.
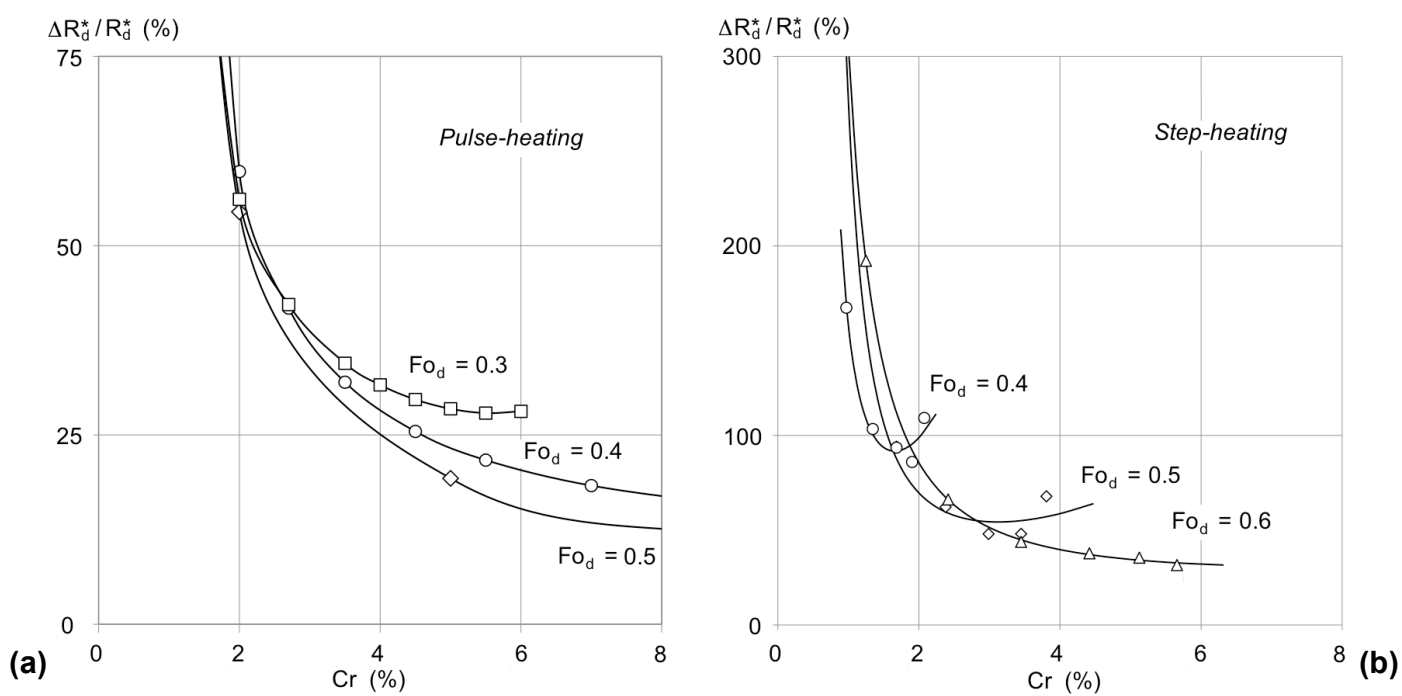

Fig. 7. Intrinsic accuracy of the identified thermal resistance of the defect using the defect-depth already identified from extrapolation to zero-contrast: (a) pulse-heating, taken from [3]; (b) step-heating, using Eq. (16). 
The results presented in Fig. $7 \mathrm{~b}$ show that the errors on $R_{d}^{*}$ are:

- $\quad$ much higher than those on the defect depth (roughly by a factor of 4, as seen in Fig. 6);

- $\quad$ more important than the errors on $R_{d}^{*}$ identified by the pulse technique (given in Fig. 7a).

Moreover, it is shown that the identification must be done for contrast values not too low (typically higher than $1.5 \%$ ), in order to guarantee errors not exceeding $100 \%$.

Figure 8 presents the same results as the ones of Figure $7 \mathrm{~b}$ under a more practical way, the error $\Delta R_{d}^{*} / R_{d}^{*}$ being given as a function of the directly measured parameters: time and contrast.

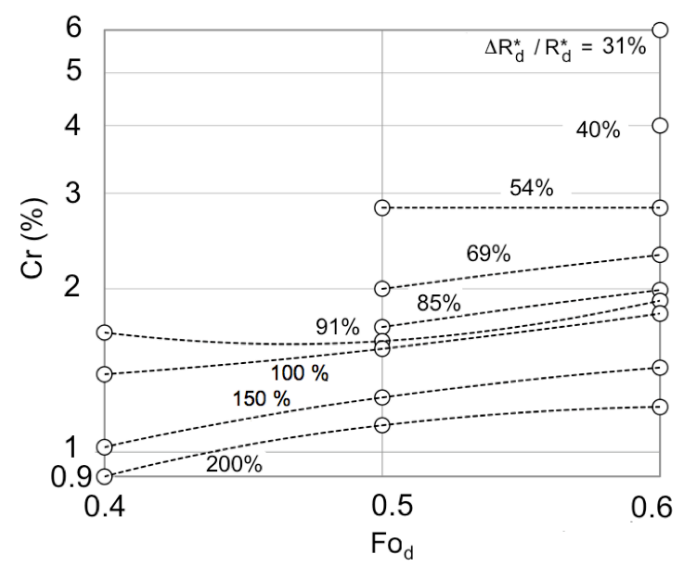

Fig. 8. Step-heating intrinsic accuracy of the identified thermal resistance of the defect using the defect-depth already identified from extrapolation to zero-contrast: presentation as a function of the Fourier number and the thermal contrast.

Finally, once the thermal resistance is identified, it is possible to estimate the error on the identified defect depth using Eq. (14), $\left(\Delta z_{d}^{*} / z_{d}^{*}\right)_{C r \rightarrow 0}$. The subtraction of this estimated error to the initially identified defect depth improves the accuracy of the latter, and the so-corrected value of $z_{d}$ can be introduced in Eq. (17), leading to a better estimate of the thermal resistance.

\subsection{Discussion}

The intrinsic accuracy of the proposed identification has been evaluated and compared to the one reached with a pulse-heating excitation. The biases on the identified defect depth and thermal resistance are respectively 2.2 times and 4 times higher for step-heating than for pulse-heating.

A correction of the bias of the defect depth is proposed, which notably mitigates both depth and thermal resistance final biases. The improvements have not been evaluated because that would be of no real use since other errors, linked to signal noise and lateral heat conduction due to the limited extent of defects, are known to affect the results and may become preponderant.

\section{Concluding remarks and prospects}

The aim of the present work was to show how the tools used for front-face thermographic monitoring of pulseheating (TSR and early detection) were still relevant for step-heating.

It has been shown that the TSR operations (thermogram fitting by a logarithmic polynomial, logarithmic differentiations and subsequent detection precession) lead to analogous results for both pulse- and step-heating. This allowed us to assume that the TSR technique is applicable to step-heating and will lead to similar results: enhancement of defect detectivity by use of derivative images and all-defect imaging by the synthesis of a unique, composite image from the polynomial coefficients.

The early detection and characterization approach, based on the emerging contrast generated by defects, has been applied to step-heating. The intrinsic accuracy of the identified defect parameters (depth and thermal resistance) turns out to be not as good as the one obtained with pulse-heating. However, even though the identification of defect depth is 2.2 times less accurate than for pulse-heating, the error does not exceed a few percents. The conclusions are not as satisfactory for the thermal resistance identification, which is 10 times less accurate than for depth identification.

These theoretical results now have to be validated by experiments, with real-life issues such as noisy signals and lateral diffusion effects. This work, fully carried out in ONERA, is reported and discussed in Part II of this study [16]. 


\section{REFERENCES}

[1] Cielo P. Pulsed photothermal evaluation of layered materials. Journal of Applied Physics 1984;56 (1): $230-234$.

[2] Balageas DL, Krapez J-C, Cielo P, Pulsed photothermal modelling of layered material. Journal of Applied Physics 1986;59 (2): 348-357.

[3] Balageas DL. Defense and illustration of time-resolved pulsed thermography for NDE. Quantitative InfraRed Thermography Journal. 2012;9(1):3-32. Erratum: Quantitative InfraRed Thermography Journal. 2012;9(2):231.

[4] Balageas D, Chapuis B, Deban G, Passilly F. Improvement of the detection of defects by pulse thermography thanks to the TSR approach in the case of a smart composite repair patch. Quantitative InfraRed Thermography Journal. 2010;7(2):167187.

[5] Shepard SM. Advances in pulsed thermography. Thermosense XXIII, Proc. SPIE. 2001;4630:511-515.

[6] Shepard SM, Lhota JR, Rubadeux BA, Wang D, Ahmed T. Reconstruction and enhancement of active thermographic image sequences. Optical Engineering. 2003;42(5):1337-1342.

[7] Roche J-M, Leroy F-H., Balageas DL. Images of TSR coefficients: a simple way for a rapid and efficient detection of defects. 2013. Materials Evaluation, Vol. 72, No. 1, Jan. 2014, pp. 73-82.

[8] Krapez J-C, Balageas D. Early detection of thermal contrast in pulsed stimulated infrared thermography. Proc. Conf. QIRT 94. QIRT Open Archives: http://qirt.gel.ulaval.ca/dynamique/index.php?idD=56, paper \# QIRT 1994-039.

[9] Krapez JC, Balageas D, Deom A, Lepoutre F. Early detection by stimulated infrared thermography. Comparison with ultrasonics and holo/shearography. Advances in Signal Processing for Non destructive Evaluation of Materials. edit. X.P.V. Maldague, Kluwer Acad. Publ. NATO ASI Series E. 1994;262: 303-321.

[10] Balageas DL, In search of early time - An original approach in the thermographic identification of thermophysical properties and defects. Advances in Optical Technologies. 2013: Article ID 314906; http://dx.doi.org/10.1155/2013/314906.

[11] Aamodt LC, MacLachlan Spicer JW, Murphy JC. Analysis of characteristic thermal transit times for time-resolved infrared radiometry studies of multilayered coatings. Journal of Applied Physics. 1990;68(12):6087-6098.

[12] Maclachlan Spicer JW, Kerns WD, Aamodt LC, Murphy JC. Measurement of coating physical properties and detection of coating dibonds by time-resolved infrared radiometry. Journal of Nondestructive Evaluation. 1989;8(2):107-120.

[13] Osiander R, Spicer JWM. Time-resolved infrared radiometry with step-heating - A review. Revue Générale de Thermique.1998;37(8):680-692.

[14] Badghaish AA, Fleming DC. Non-destructive inspection of composites using step heating thermography", J. of Composite Materials, Vol. 42, No 13, pp. 1337-1357, 2008b.

[15] Dumoulin J., Ibos L., Marchetti M., Mazioud A., "Detection of non emergent defects in asphalt pavement samples by long pulse and phase infrared thermography", European Journal of Environmental and Civil Engineering, Vol. 15, pp. 557-574, 2011.

[16] Roche J.-M., Balageas D.L, Common tools for quantitative pulse and step-heating thermography - Part II: experimental validation. Submitted to Quantitative InfraRed Thermography Journal.

[17] Schenck H. Theory of engineering experimentation. 3rd ed. Co: Hemisphere Publish. 1979.

[18] Carslaw HS, Jaeger JC. Conduction of heat in solids. Oxford University Press, London. 1959:112-113.

[19] Vavilov V. Transient thermal NDT: conception in formulae. Proc. Conference QIRT 1992. QIRT Open Archives: http://www.qirt.org/dynamique/index.php?idD=55. Paper \# QIRT 1992-035.

[20] Vavilov V., Nondestructive testing handbook,. Vol. 5, Book 1: Thermal/infrared testing. Moscow: Spektr Publishing House; 2009.

[21] Balageas D., Thickness or diffusivity measurements from front-face flash experiments using the TSR (thermographic signal reconstruction) approach. QIRT 2010 Proc.: QIRT Open Archives: http://qirt.gel.ulaval.ca/dynamique/index.php?idD=50, paper \# QIRT 2010-011. 



\section{ONERA}

BP 72 - 29 avenue de la Division Leclerc - 92322 CHATILLON CEDEX - Tél. : +33 146734040 - Fax : +33 146734141

w w w. onera.f r 